\title{
Improved Performance of the Laser Guide Star Adaptive Optics System at Lick Observatory
}

\author{
S. S. Olivier, D. T. Gavel, H. W. Friedman, C. E. Max, \\ J. R. An, K. Avicola, B. J. Bauman, J. M. Brase, \\ E. W. Campbell, C. Carrano, J. B. Cooke, G. J. Freeze, \\ E. L. Gates, V. K. Kanz, T. C. Kuklo, B. A. Macintosh, \\ M. J. Newman, E. L. Pierce, K. E. Waltjen and J. A. Watson
}

This paper was prepared for submittal to the 44th Annual Meeting of the International Symposium on

Optical Science, Engineering, and Instrumentation.

Denver, Colorado

July 18-23, 1999

July 20, 1999

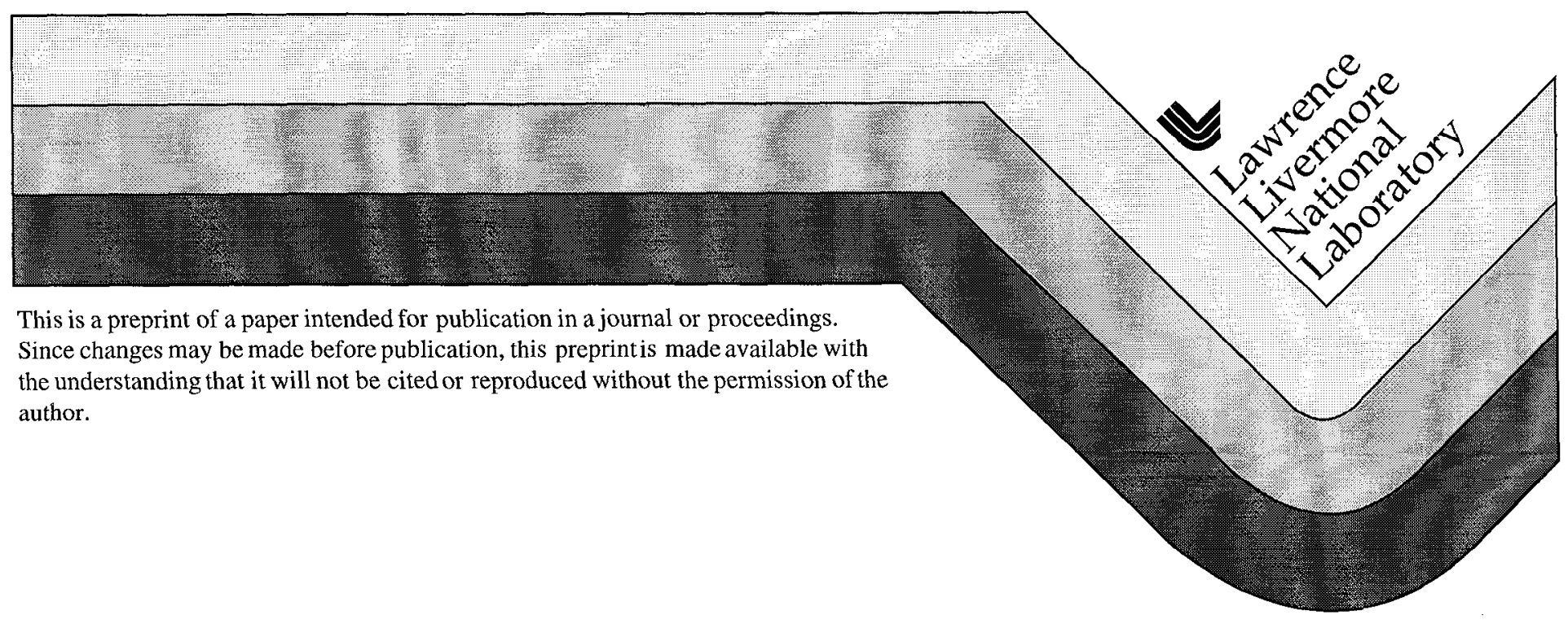




\section{DISCLAIMER}

This document was prepared as an account of work sponsored by an agency of the United States Government. Neither the United States Government nor the University of California nor any of their employees, makes any warranty, express or implied, or assumes any legal liability or responsibility for the accuracy, completeness, or usefulness of any information, apparatus, product, or process disclosed, or represents that its use would not infringe privately owned rights. Reference herein to any specific commercial product, process, or service by trade name, trademark, manufacturer, or otherwise, does not necessarily constitute or imply its endorsement, recommendation, or favoring by the United States Government or the University of California. The views and opinions of authors expressed herein do not necessarily state or reflect those of the United States Government or the University of California, and shall not be used for advertising or product endorsement purposes. 


\title{
Improved performance of the laser guide star adaptive optics system at Lick Observatory
}

\author{
Scot S. Olivier ${ }^{*}$, Donald T. Gavel, Herbert W. Friedman, Claire E. Max, Jong R. An, Kenneth Avicola, \\ Brian J. Bauman, James M. Brase, Eugene W. Campbell, Carmen Carrano, Jeffrey B. Cooke, Gary J. \\ Freeze, Elinor L. Gates ${ }^{a}$, Vernon K. Kanz, Thomas C. Kuklo, Bruce A. Macintosh, Michael J. Newman, \\ Edward L. Pierce, Kenneth E. Waltjen, James A. Watson \\ Lawrence Livermore National Laboratory, PO Box 808, Livermore, CA 94551 \\ ${ }^{a}$ Lick Observatory, Mt. Hamilton, CA 95140
}

\begin{abstract}
Results of experiments with the laser guide star adaptive optics system on the 3-meter Shane telescope at Lick Observatory have demonstrated a factor of 4 performance improvement over previous results. Stellar images recorded at a wavelength of $2 \mu \mathrm{m}$ were corrected to over $40 \%$ of the theoretical diffraction-limited peak intensity. For the previous two years, this sodium-layer laser guide star system has corrected stellar images at this wavelength to $\sim 10 \%$ of the theoretical peak intensity limit. After a campaign to improve the beam quality of the laser system, and to improve calibration accuracy and stability of the adaptive optics system using new techniques for phase retrieval and phase-shifting diffraction interferometry, the system performance has been substantially increased. The next step will be to use the Lick system for astronomical science observations, and to demonstrate this level of performance with the new system being installed on the 10-meter Keck II telescope.
\end{abstract}

Keywords: adaptive optics, laser guide star, sodium layer, imaging

\section{INTRODUCTION}

Images of astronomical objects produced by ground-based telescopes are blurred by the Earth's atmosphere. Adaptive optics can correct for these atmospheric aberrations and provide images with resolution limited only by diffraction from the telescope aperture.' An adaptive optics system requires a reference source to sense the atmospheric aberrations. The reference source can be a natural star, but requirements on the brightness and proximity of the reference source limit the fraction of the sky accessible with natural stars to a few percent for near-infra-red imaging. Use of a laser guide star can increase the available fraction of the sky since the laser can be directed to any desired location. With a laser guide star the sky coverage is limited only by the need to use a natural star to control overall image motion. Image motion cannot be controlled by the laser guide star since its position is randomly varied during propagation up through the atmosphere. Since the requirements on the brightness and proximity of the reference source for the control of image motion are less severe than for full correction of the atmospheric aberrations, use of a laser guide star increases the available fraction of the sky by a factor of -10 .

A laser guide star adaptive optics system has been developed for the 3-meter Shane telescope at the University of California's Lick Observatory, located on Mt. Hamilton near San Jose, California. The laser guide star is formed by resonance fluorescence of atomic sodium located in a layer near the top of the mesosphere at $\sim 95 \mathrm{~km}$ altitude. This sodium-layer laser guide star provides better sampling of the atmospheric turbulence for large telescopes than a guide star formed by Rayleigh scattering from air molecules at lower altitudes, $5-20 \mathrm{~km}$.

The Lick Observatory laser guide star adaptive optics system first produced significant image correction in September $1996 .^{2}$ In this paper, we describe the results of experiments with the Lick laser guide star adaptive optics system conducted during November 1998.

\footnotetext{
* Correspondence: email: olivier1@llnl.gov; www: http://ep.llnl.gov/urp/science/lgs_www/lgs.html; phone: 925-423-6483; fax: 925-422-1796
} 


\section{SYSTEM OVERVIEW}

\subsection{Adaptive optics system}

The Lick adaptive optics system ${ }^{3}$ is mounted at $\mathrm{f} / 17$ Cassegrain focus of 3 -meter Shane Telescope. The system uses a deformable mirror built by LLNL with 127 actuators arranged in a triangular pattern. The central 61 actuators are actively controlled. A separate flat fast steering mirror built by Physik Instrumente is used to control overall image motion. The wavefront sensor is a Shack-Hartmann design with 40 active sub-apertures (44-cm diameter mapped to the telescope primary) arranged in a square pattern. The wavefront sensor camera built by camera Adaptive Optics Associates has a frame rate of up to $1.2 \mathrm{kHz}$, and uses a $64 \times 64 \mathrm{CCD}$ built by Lincoln Labs with $7 \mathrm{e}^{-}$read noise. A separate quad-cell tip-tilt sensor built by LLNL using photon-counting avalanche photo-diodes built by EG\&G can be used for image motion control with a natural star when the wavefront sensing is done with the laser guide star. The control computer is a 160-Mflop model built by Mercury with 4 Intel i 860 processors.

In September 1996, the Lick AO system had over $300 \mathrm{~nm}$ rms of residual error due to instrumental calibration. These errors were dominated by aberrations due to alignment of the infra-red camera. New calibration techniques have improved control of instrumental errors in Lick adaptive optics system. Two high-accuracy in-situ calibration techniques have been demonstrated: phase diverse phase retrieval ${ }^{1}$ with an accuracy of $\sim 20 \mathrm{~nm} \mathrm{rms}$, and phase shifting diffraction interferometry ${ }^{5}$ with an accuracy of $\sim 10 \mathrm{~nm}$ rms. Using these techniques, the instrumental errors have been reduced to the $100 \mathrm{~nm}$ rms level. The remaining instrumental error sources include deformable mirror surface quality ( $50 \mathrm{~nm}$ rms), non-common path air turbulence $(-50 \mathrm{~nm} \mathrm{rms})$, and non-common path flexure $(\sim 70 \mathrm{~nm} \mathrm{rms})$. The instrumental error can be further reduced by additional improvement of the system opto-mechanical stability, automation of the calibration procedures to provide periodic compensation of non-common path flexure during operation, reduction of the non-common path length, improved control of air flow in the system, and installation of a new deformable mirror with better surface quality. Several of these issues are being addressed in 1999 with an improved opto-mechanical design for the Lick adaptive optics system. ${ }^{6}$

\subsection{Laser System}

The Lick laser system ${ }^{7}$ is based on a pulsed dye laser capable of up to $20 \mathrm{~W}$ average power output that is tuned to the $589 \mathrm{~nm}$ sodium $\mathrm{D}_{2}$ resonance line. A set of four flash-lamp pumped frequency-doubled solid-state (Nd:YAG) pump lasers are located in a room below the dome floor, and fiber-optics relay the pump light to the dye laser mounted on the telescope. Beam control is achieved with active pointing and centering loops. A wavefront sensor and a far-field camera are used to assess beam quality. A fast up-link tip-tilt control system is used to stabilize the image of the laser guide star in the adaptive optics wavefront sensor. The beam is launched from a $30 \mathrm{~cm}$ refractive telescope.

The Lick laser system is typically operated with an output power of $15 \mathrm{~W}$ (nearly always between 13 to $18 \mathrm{~W}$ ) at $589 \mathrm{~nm}$. The return signal varies from month to month, ranging from $\sim 9.5$ to $\sim 7.0$ equivalent $\mathrm{V}$ magnitude. The maximum and minimum values have been observed in multiple months during the past 4 years of operation. Variation in the laser output power and wavelength tuning are likely to account for less than a factor of 2 the observed variation in return signal. Therefore, the sodium density appears to vary by a factor of $\sim 5$.

The performance of the adaptive optics system is strongly affected by the size of guide star. Theoretically, the error in the measurement of the wavefront scales linearly with angular size of the reference source as seen by the wavefront sensor subapertures. The scaling of error with spot size can become more severe due to physical limits in the AO system such as the field of view of the wavefront sensor subapertures. If a significant fraction of light from the guide star begins to fall outside of the field of view, the wavefront measurement error rises rapidly.

In 1998, the laser guide star spot size was improved by changing the dye amplifier configuration so that the beam bounces as it passes through the dye cell. ${ }^{8}$ This configuration was previously tested in a laser system built by LLNL for the Keck Observatory. ${ }^{9}$ The new configuration improved the $80 \%$ enclosed-energy diameter from 6.6 arc seconds in September 1997 to 4.2 arc seconds in November 1998. The current spot size is apparently limited by aberrations introduced in the alignment of the launch telescope. For this system, a beam that is 1.5 times diffraction-limited would have an $80 \%$ enclosed energy diameter less than 3 arc seconds in good ( 0.75 arc second) seeing. This should be achievable with better launch telescope alignment. 


\section{TEST RESULTS}

Figure 1 shows images of point sources obtained with the Lick adaptive optics system on November 12, 1998. Figure 1a shows correction of 59 Andromeda (SAO55331) to a Strehl ratio of 0.65 at a wavelength of $2 \mu$ In using this $R \approx 7$ natural guide star as the wavefront reference source. Similar performance was also achieved with dimmer stars $(\mathrm{R}<10)$. Figure $1 \mathrm{~b}$ shows correction of a field star near SAO38239 to a Strehl ratio of 0.42 at a wavelength of $2 \mu \mathrm{m}$ using the laser guide star as the wavefront reference source and this $R \approx 12$ natural star as the tip-tilt reference source.

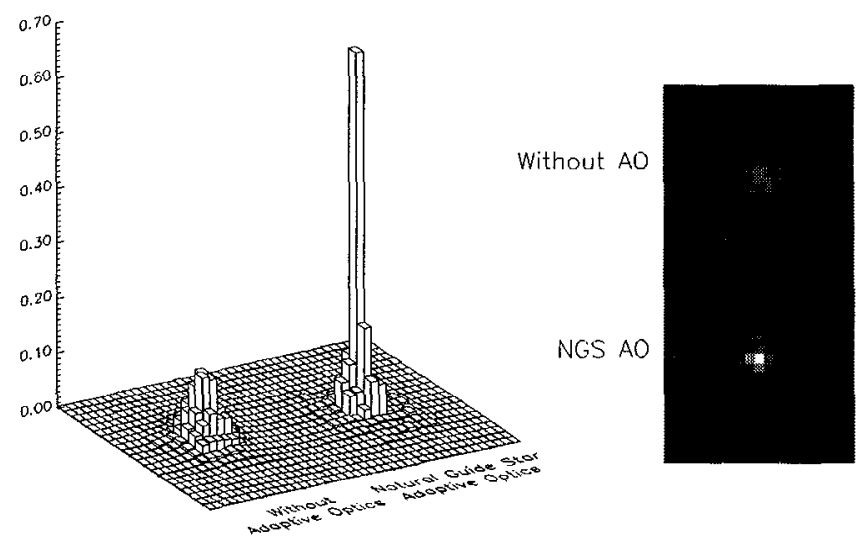

Figure 1a. Natural guide star adaptive optics corrected image of 59 Andromeda. Strehl ratio $=0.65$ at a wavelength of $2 \mu \mathrm{m}$

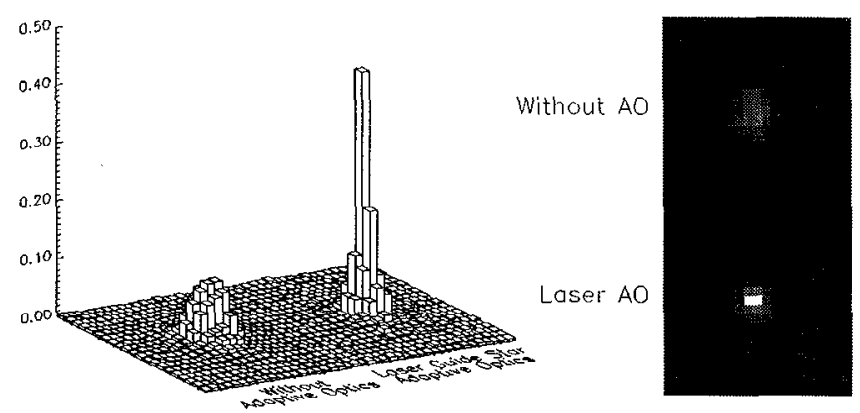

Figure 1b. Laser guide star adaptive optics corrected image of an $\mathrm{R} \approx 12$ field star near $\mathrm{SAO} 38239$. Strehl ratio $=$ 0.42 at a wavelength of $2 \mu \mathrm{m}$

Estimates of the residual errors for the corrected images using the natural and laser guide stars are shown in Table 1. "Fitting error" is due to the inability of the deformable mirror to respond to high-spatial-frequency variations of the atmosphere. "Servo error" is due to the inability of the control loop to respond to the high-temporal-frequency variations of the atmosphere. "Measurement error" is due to the inability of the wavefront sensor to accurately measure the reference wavefront in the presence of noise. "Calibration error" is due to residual non-common-path aberration between the wavefront sensor and science camera focal planes. "Cone effect" measures the difference between the wavefront measured from the finite-altitude laser guide star and from a real star located an "infinite" distance away.

Table 1. Residual wavefront errors after adaptive optics correction

\begin{tabular}{lll}
\hline Residual error source & NGS AO error & LGS AO error \\
\hline Fitting & $97 \mathrm{~nm} \mathrm{rms}$ & $97 \mathrm{~nm} \mathrm{rms}$ \\
Servo & $97 \mathrm{~nm} \mathrm{rms}$ & $145 \mathrm{~nm} \mathrm{rms}$ \\
Measurement & $8 \mathrm{~nm} \mathrm{rms}$ & $171 \mathrm{~nm} \mathrm{rms}$ \\
Calibration & $100 \mathrm{~nm} \mathrm{rms}$ & $100 \mathrm{~nm} \mathrm{rms}$ \\
Cone & & $55 \mathrm{~nm} \mathrm{rms}$ \\
& & $269 \mathrm{~nm} \mathrm{rms}$ \\
Total & $168 \mathrm{~nm} \mathrm{rms}$ & \\
& & 0.49 \\
Predicted Strehl $(2 \mu \mathrm{m})$ & 0.76 & 0.42 \\
Measured Strehl $(2 \mu \mathrm{m})$ & 0.65 & \\
\hline
\end{tabular}

The predicted Strehl ratios given in Table 1 are approximated by $\exp \left(-\sigma^{2}\right)$ where $\sigma$ is the rms wavefront error in radians at 2 $\mu \mathrm{m}$. The measured Strehl ratios were calculated by computing the diffraction-limited point spread function, resampling at the infra-red camera plate scale ( 0.125 arc seconds per pixel), and shifting to give the best fit to the measured image. The 
fraction of the light within the brightest pixel in the measured image is then compared to the fraction within the brightest pixel in the synthetic image and extrapolated to deduce the on-axis Strehl.

The fitting error was estimated using Monte Carlo simulations of the atmosphere and a deformable mirror model based on interferometric measurements of the actuator influence functions. The results were scaled to correspond to the observed seeing of 0.5 arc second. Measurement and servo errors were determined directly from measured wavefront phase power spectra using diagnostic data from the wavefront sensor. The measurement error was estimated from the integral of the power spectrum noise floor, taking into account filtering of the noise by the control loop. Servo error was calculated from the integral of the power spectrum after removal of the noise floor. Calibration error was measured using images of an internal white-light reference point source. The cone effect was estimated based on a theoretical model of the atmosphere scaled to the observed seeing. The difference in the predicted and measured Strehl ratios in Table 1 is due in part to residual tip-tilt error, which has not been included in this analysis.

\section{FUTURE PLANS}

Based on the improved performance obtained in 1998, the Lick adaptive optics system is being upgraded in 1999 to be useable as a facility-class instrument. The upgrades include an improved opto-mechanical design, automated target acquisition and automated calibration. In addition, Lick Observatory has hired staff to support operation of the adaptive optics system. The infra-red camera used with the adaptive optics system has also been upgraded to a new diffraction-limited camera built under the direction of U.C. Berkeley Prof. James Graham. This camera also has the capability for lowresolution spectroscopy with $\Delta \lambda \lambda \approx 1000$. Designs for a high-resolution spectrograph are currently being studied. ${ }^{10}$ Following the upgrade of the adaptive optics system, the laser system will be upgraded in 2000-2001 to be useable as a facility-class instrument. These upgrades include reconfiguring the pump lasers into a single rack-mounted unit, and automating the diagnostics and controls for beam alignment.

Along with the upgrades to the instrumentation, the Lick laser guide star adaptive optics system will be used for astronomy by University of California faculty, staff, and students. Several projects have already hegun using natural guide stars. These projects include studies of young stars, solar system planets and companion searches. Figure 2 shows images of Neptune and Triton obtained for one of the ongoing astronomy projects. The first astronomy paper using Lick adaptive optics data to set limits on possible stellar companions to a nearby star with a detected planetary companion in a highly eccentric orbit has been published. ${ }^{11}$

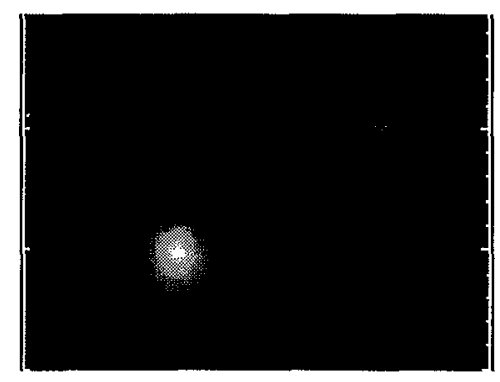

Uncorrected

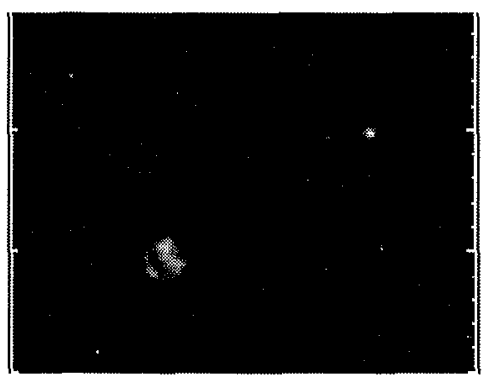

Corrected

Figure 2. Images of Neptune and Triton obtained with the Lick adaptive optics system. Corrected images allow the study of Neptune's atmosphere.

Astronomy projects using the laser guide star are also now beginning. These projects include studies of active galactic nuclei and young stars. Figure 3 shows images of the young star FS Tauri $(R=15)$ obtained for one of these projects.

The Lick laser guide star adaptive optics system has also served as a prototype for the system at Keck Observatory that was built collaboratively by Keck Observatory and LLNL. ${ }^{12}$ The Keck adaptive optics system produced corrected images during the first engineering tests in February 1999. Images from the first engineering and astronomy demonstration tests can be seen 
on the World Wide Web at http://www2.keck.hawaii.edu:3636/realpublic/ao/aolight.html. The next step in the development of the Keck system will be for Keck and LLNL to collaborate on the installation of the LLNL-built laser system in 2000.

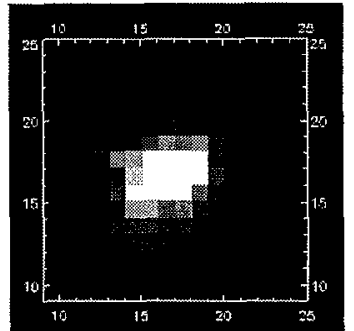

Uncorrected

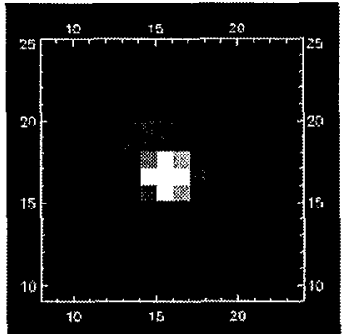

Corrected

Figure 3. Images of FS Tauri obtained with the Lick laser guide star adaptive uplics system. The known companion at a separation of 0.24 arc seconds can be seen in the corrected image. The corrected image can be used to provide infra-red photometry for this binary pair to constrain models.

\section{CONCLUSION}

Performance of the Lick Observatory laser guide star adaptive optics system was improved in 1998. The best corrected images using the sodium-layer laser guide star achieved a Strehl ratio of 0.42 at a wavelength of $2 \mu \mathrm{m}$. Images corrected using natural guide stars achieved Strehl ratios up to 0.65 at a wavelength of $2 \mu \mathrm{m}$. These results, and the residual error models that explain the results, assure us that there are no unanticipated systematic errors preventing adaptive optics astronomy with a sodium laser guide star.

The Lick adaptive optics system performance when using the laser guide star is currently limited by the laser guide star spot size. When using bright natural guide stars, the system performance is limited by the accuracy of the internal calibration for non-common path aberrations. Efforts to continue to improve both the laser guide star spot size and the adaptive optics system calibration are currently underway as part of an overall effort to upgrade the Lick laser guide star adaptive optics system to a facility-class instrument.

\section{ACKNOWLEDGEMENTS}

Work performed under the auspices of the U.S. Department of Energy by the Lawrence Livermore National Laboratory under Contract number W 7405-Eng-48.

\section{REFERENCES}

1. H. W. Babcock, "Adaptive optics revisited", Science 249, pp. 253-257, 1990.

2. C. E. Max, S. S. Olivier, H. W. Friedman, J. An, K. Avicola, B. V. Beeman, H. D. Bissinger, J. M. Brase, G. V. Erbert, D. T. Gavel, K. Kanz, M. C. Liu, B. Macintosh, K. P. Neeb, J. Patience, and K. E. Waltjen, "Image improvement from a sodium-layer laser guide star adaptive optics system", Science 277, pp. 1649-1652, 1997.

3. J. M. Brase, J. An, K. Avicola, H. D. Bissinger, H. W. Friedman, D. T. Gavel, B. Johnston, C. E. Max, S. S. Olivier, R. Presta, D. A. Rapp, J. T. Salmon, and K. E. Waltjen, "Adaptive optics at Lick Observatory: System architecture and operations", Proc. SPIE 2201, pp. 474-488, 1994.

4. C. J. Carrano, S. S. Olivier, J. M. Brase, B. A. Macintosh, and J. R. An, "Phase retrieval techniques for adaptive optics", Proc. SPIE 3353, pp. 658-667, 1998.

5. E. W. Campbell, B. J. Bauman, D. R. Sweider, and S. S. Olivier, "High-accuracy calibration of an adaptive optics systern using a phase shifting diffraction interferometer", Proc. SPIE 3762, in press, 1999. 
6. B. J. Bauman, D. T. Gavel, K. E. Waltjen, G. J. Freeze, K. A. Keahi, T. C. Kuklo, S. K. Lopes, M. J. Newman, S. S. Olivier, "New optical design of adaptive optics system at Lick Observatory", Proc. SPIE 3762, in press, 1999.

7. H. W. Friedman, G. V. Erbert, T. C. Kuklo, J. G. Malik, J. T. Salmon, D. A. Smauley, G. R. Thompson, and N. J. Wong, "Sodium beacon laser system for the Lick Observatory", Proc. SPIE 2534, pp. 150-160, 1995.

8. D. T. Gavel, H. W. Friedman, and B. J. Bauman, "Lick sodium laser guide star: performance during the 1998 LGS observing campaign", Proc. SPIE 3762, in press, 1999.

9. H. W. Friedman, J. B. Cooke, P. M. Danforth, G. V. Frhert, M. Feldman, D. T. Gavel, S. I. Ienkins, H. E. Jones, V. K. Kanz, T. Kuklo, M. J. Newman, E. L. Pierce, R. W. Presta, J. T. Salmon, G. R. Thompson, and N. J. Wong, "Design and performance of a laser guide star system for the Keck II telescope", Proc. SPIE 3353, pp. 260-276, 1998.

10. J. Ge, J. P. Angel, R. Q. Fugate, C. E. Max, P. J. Kuzmenko, D. R. Ciarlo, D. W. McCarthy, Jr., N. J. Woolf, M. LloydHart, S. S. Olivier, K. H. Cook, J. Najita, "Adaptive optics high-resolution spectroscopy: present status and future direction", Proc. SPIE 3762, in press, 1999.

11. G. W. Marcy, R. P. Butler, S. S. Vogt, D. Fischer, and M. C. Liu, "Two New Candidate Planets in Eccentric Orbits" Astrophys. J. 520, pp. 239-247, 1999.

12. P. Wizinowich, D. S. Acton, T Gregory, P. Stomski, J. An, K. Avicola, J Brase, H. Friedman, D. Gavel, C. Max, "Status of the W. M. Keck Observatory Adaptive Optics Facility", Proc. SPIE 3353, pp. 568-578, 1998. 